\title{
Enteral $n-3$ fatty acids and micronutrients enhance percentage of positive neutrophil and lymphocyte adhesion molecules: a potential mediator of pressure ulcer healing in critically ill patients
}

\author{
Miriam Theilla ${ }^{1,2}$, Betty Schwartz ${ }^{2}$, Yael Zimra ${ }^{3}$, Haim Shapiro ${ }^{1}$, Ronit Anbar ${ }^{1}$, Esther Rabizadeh ${ }^{3}$, \\ Jonathan Cohen ${ }^{1}$ and Pierre Singer ${ }^{1 *}$ \\ ${ }^{1}$ Department of General Intensive Care, Institute for Nutrition Research, Rabin Medical Center, Campus Beilinson, Petah \\ Tikva 49100, Israel \\ ${ }^{2}$ The Robert H. Smith Faculty of Agriculture, Food and Environment, The Hebrew University, Jerusalem, Israel \\ ${ }^{3}$ Department of Hematology, Rabin Medical Center, Petab Tikva 49100, Israel
}

(Submitted 19 October 2010 - Final revision received 23 June 2011 - Accepted 24 June 2011 - First published online 1 November 2011)

\section{Abstract}

$n-3$ Fatty acids are recognised as influencing both wound healing and immunity. We assessed the impact of a fish oil- and micronutrientenriched formula (study formula) on the healing of pressure ulcers and on immune function in critically ill patients in an intensive care unit. A total of forty patients with pressure ulcers and receiving nutritional support were enrolled (intervention group, $n$ 20, received study formula; and a control group, $n$ 20, received an isoenergetic formula). Total and differential leucocyte count and percentage of adhesion molecule positive granulocyte and lymphocyte cells (CD11a, CD11b, CD18 and CD49b) were measured on days 0, 7 and 14 . Percentage of positive lymphocytes for CD54, CD49b, CD49d and CD8 were also measured on days 0,7 and 14 . The state of pressure ulcers was assessed by using the pressure ulcer scale for healing tool score on days 7, 14 and 28 of treatment. No between-group differences in patient demographics, anthropometry or diagnostic class were observed. Patients who received the study formula showed significant increases in the percentage of positive CD18 and CD11a lymphocytes and of CD49b granulocytes as compared to controls $(P<0 \cdot 05)$. While the severity of pressure ulcers was not significantly different between the two groups on admission, severity increased significantly over time for the control group $(P<0 \cdot 05)$, but not for the study group. The present study suggests that a fish oil- and micronutrient-enriched formula may prevent worsening of pressure ulcers and that this effect may be mediated by an effect on adhesion molecule expression.

Key words: $n$-3 Fatty acids: Pressure ulcers: Adhesion molecules

Wound healing is a complex, multi-stage process by which tissue integrity is restored following injury or infection. The process comprises a series of events, including a coagulation phase, an inflammatory phase and a final repair phase. The inflammatory phase plays an essential role in preventing infection, as also an equally essential role in aiding in the tissue repair process. This physiological repair response requires a complex and dynamic interplay of several cell types, including local tissue and recruited haematopoietic cells. Blood cell migration into the wound involves attachment to the endothelial cells and extravasation, a process which is regulated mainly by chemokines and cellular adhesion molecules (CAM). These CAM, integrins and selectins, direct leucocyte-endothelium interactions, transendothelial migration of leucocytes and leucocyte trafficking in general ${ }^{(1)}$. Their importance in wound healing was clearly demonstrated by Cruse \& Wang $^{(2)}$ who showed that delayed healing of pressure ulcers in spinal cord injury patients could be attributed to reduced CAM expression, impaired cell-cell interaction and lack of extracellular matrix structural and functional protein.

Among other compounds, lipids, especially the $n-3$ longchain PUFA ( $n$-3-LC-PUFA) have been shown to influence the immune response, and therefore have an effect on wound healing. Thus, PUFA deficiency has been shown to result in impaired wound healing in patients ${ }^{(3)}$, as well as in rats, who received an essential fatty acid-deficient diet ${ }^{(4)}$. On the other hand, we have previously demonstrated that the administration of an enteral nutrition formula enriched in PUFA and micronutrients reduced the incidence of new pressure ulcers in patients in an intensive care unit (ICU) with acute lung injury ${ }^{(5)}$. There are, however, few data regarding the

Abbreviations: CAM, cellular adhesion molecules; ICU, intensive care unit; $n$-3-LC-PUFA, $n$-3 long-chain PUFA.

* Corresponding author: Professor P. Singer, fax +972 39232333 , email psinger@clalit.org.il 
possible role of immune function in the healing of pressure ulcers in critically ill patients.

The objective of the present clinical trial was to assess the impact of a fish oil- and micronutrient-enriched formula on the expression of adhesion molecules on peripheral blood leucocytes and to correlate this with the healing of pressure ulcers in critically ill patients.

\section{Materials and methods \\ Patients}

This prospective, controlled, randomised study was conducted according to the guidelines laid down in the Declaration of Helsinki and all procedures involving human subjects were approved by the Rabin Medical Center Review Board. Written informed consent was obtained from all patients or from a legal guardian where relevant. Adult patients admitted to the general ICU were considered eligible for enrolment if: (1) they presented with or developed greater than or equal to one grade II (or higher) pressure ulcer/s (i.e. damage to the epidermis extending at least into the dermis) according to the National Pressure Ulcer Advisory Panel classification ${ }^{(6)}$ and (2) they were expected to be in need of nutritional support for at least $5 \mathrm{~d}$. Patients were excluded if there was evidence of pre-existing impaired wound healing or abnormal immune status, e.g. patients receiving chemotherapy or treatment with more than $0.25 \mathrm{mg} / \mathrm{kg}$ per d prednisone (or an iso-equivalent dose of other glucocorticoids). Significant intracranial haemorrhage was also an exclusion criterion.

\section{Nutritional support}

Patients were randomly allocated to receive either fish oiland micronutrient-enriched enteral nutrition (Oxepa; Abbott, Chicago, IL, USA - the intervention group) or an isonitrogenous formula (Jevity; Abbott - the control group) according to a computer-generated random list in the ratio of 1:1. The macro- and micronutrient composition of the various nutritional formulae is depicted in Table 1 . There was no significant difference in the mean protein intake over the study period between the two groups $(66.1 \mathrm{~g} / \mathrm{d}$ for the study group $v \cdot 65 \cdot 1 \mathrm{~g} / \mathrm{d}$ for the control group). The study statistician was blinded to the treatment allocation, but not the ICU staff, patients or assessor of ulcer severity. The quantity of nutritional formula prescribed was determined by the measurement of resting energy expenditure as assessed by indirect calorimetry (Deltatrac II; Datex-Ohmeda, Helsinki, Finland), which is the standard of care in our ICU. Resting energy expenditure was assessed every $7 \mathrm{~d}$ and the energy prescription adjusted accordingly. Where patients were unable to receive the full energy prescription via the enteral route, e.g. due to gastric paresis, enteral nutrition was supplemented with parenteral nutrition. All other aspects of patient management, including local ulcer treatment were determined by the patients' attending physician.
Table 1. Composition of selected nutrients in the control and study formulae per $100 \mathrm{ml}$

\begin{tabular}{|c|c|c|c|c|}
\hline \multirow[b]{2}{*}{ Nutrient } & \multicolumn{2}{|c|}{ Control formula } & \multicolumn{2}{|c|}{ Intervention formula } \\
\hline & Enteral $^{*}$ & Parenteral† & Enteral‡ & Parenteral§ \\
\hline $\mathrm{CHO}(\mathrm{g})$ & $15 \cdot 4$ & $14 \cdot 1$ & $10 \cdot 5$ & $14 \cdot 1$ \\
\hline Fat $(\mathrm{g})$ & 3.5 & $4 \cdot 0$ & $9 \cdot 4$ & $4 \cdot 0$ \\
\hline Protein (g) & $4 \cdot 4$ & $3 \cdot 4$ & $6 \cdot 2$ & $3 \cdot 4$ \\
\hline EPA (g) & 0 & 0 & 0.46 & $0.125-0.282$ \\
\hline $\mathrm{DHA}(\mathrm{g})$ & 0 & 0 & 0 & $0.144-0.309$ \\
\hline GLA (g) & 0 & 0 & 0.4 & 0 \\
\hline Vitamin C (mg) & $15 \cdot 7-22 \cdot 5$ & 125 & 850 & 125 \\
\hline Vitamin E (IU) & $2 \cdot 3-3 \cdot 4$ & $1 \cdot 12$ & $32 \cdot 0$ & $1 \cdot 12$ \\
\hline Vitamin A (IU) & 375.9 & $350 \cdot 0$ & $667 \cdot 8 \|$ & $350 \cdot 0$ \\
\hline $\mathrm{Cu}(\mathrm{mg} / 1000 \mathrm{ml})$ & $1-1.5$ & 0.4 & $2 \cdot 2$ & 0.4 \\
\hline $\mathrm{Mn}(\mathrm{mg} / 1000 \mathrm{ml})$ & $2 \cdot 6-3 \cdot 7$ & 0.1 & $5 \cdot 3$ & 0.1 \\
\hline $\mathrm{Zn}(\mathrm{mg} / 1000 \mathrm{ml})$ & $16 \cdot 8$ & 1 & $23 \cdot 9$ & 1 \\
\hline
\end{tabular}

$\mathrm{CHO}$, carbohydrates; GLA, $\gamma$-linolenic acid.

* Jevity (Abbott, Chicago, IL, USA).

† OliClinomel N-6 990 E (Baxter, Deerfield, IL, USA)

$\ddagger$ Oxepa (Abbott).

$\S$ OliClinomel N-6 990 E (Baxter) and Omegaven (Fresenius Kabi, Bad Hamburg, Germany).

\| Supplied by $5 \mathrm{mg} \beta$-carotene.

\section{Measurement of serum parameters}

On admission, the following were recorded: patient age, sex, BMI, primary diagnosis (surgical, medical or trauma) and Acute Physiology and Chronic Health Evaluation II score ${ }^{(7)}$. The latter provides both an estimate of ICU mortality and is inversely correlated to reactive skin hyperaemia ${ }^{(8)}$. Total and differential peripheral leucocyte counts were assessed as part of the routine full blood count performed daily in the ICU.

\section{Measurement of percentage of positive adhesion molecules}

Blood was drawn from an indwelling venous catheter on days 0, 7 and 14. Patients continued to receive nutritional support before blood withdrawal. Sodium heparin-preserved whole blood was stained with the following fluorescein isothiocyanate isomer 1 - or phycoerythrin - conjugated monoclonal antibodies, in preparation for flow cytometric analysis: CD11a, CD11b, CD18, CD49b, CD49d, CD54, CD8, CD44 (Table 2$)$. Whole blood $(100 \mu \mathrm{l})$ was combined with $20 \mu \mathrm{l}$ of the fluorescent conjugated antibodies and incubated in the dark at room temperature for $10 \mathrm{~min}$. A cell-suspension was then prepared using a Coulter Q-Prep, which contained an erythrocyte lytic agent and a leucocyte stabiliser. The samples were washed and suspended in $1 \mathrm{ml}$ of formalin fixative. All samples were analysed using the Coulter Epics XL Flow Cytometer Analyzer (Beckman Coulter, Fullerton, CA, USA). Lymphocyte and granulocyte gates were set for the measurement of positively stained lymphocytes and granulocytes.

\section{Assessment of pressure ulcer severity}

The diagnosis of a grade II pressure ulcer before enrolment and monitoring of changes in ulcer severity were assessed by the Pressure Ulcer Scale for Healing tool score ${ }^{(9)}$, a non-invasive clinical aid that attributes a severity score to 
Table 2. Designation(s), expression and role of cellular adhesion molecules measured

\begin{tabular}{|c|c|c|c|c|c|}
\hline Family & $\begin{array}{c}\mathrm{CD} \\
\text { designation }\end{array}$ & $\begin{array}{l}\text { Other name (s), } \\
\text { description }\end{array}$ & Expressed by & Ligand(s) & Function(s) \\
\hline \multirow[t]{10}{*}{ Integrins } & CD11a & $\alpha$-Chain of LFA-1 & & & \\
\hline & CD11b & $\alpha$-Chain of Mac-1 & & & \\
\hline & CD18 & $\begin{array}{l}\beta \text {-Chain of LFA-1, } \\
\text { Mac-1 }\end{array}$ & & & \\
\hline & CD11a/CD18 & LFA-1, integrin $\alpha \mathrm{L} \beta 2$ & $\begin{array}{l}\text { Monocyte/macrophage, } \\
\text { neutrophils, T cells, B cells }\end{array}$ & ICAM-1 & $\begin{array}{l}\text { Firm leucocyte-endothelium } \\
\text { adhesion, trans-migration, signal } \\
\text { transduction, immunologic } \\
\text { synapse formation }\end{array}$ \\
\hline & $\mathrm{CD} 11 \mathrm{~b} / \mathrm{CD} 18$ & $\begin{array}{l}\text { Mac-1, CR3, integrin } \\
\alpha \mathrm{M} \beta 2\end{array}$ & $\begin{array}{l}\text { Monocyte/macrophage, } \\
\text { neutrophils, T cells, B cells }\end{array}$ & ICAM-1, iC3b & $\begin{array}{l}\text { Firm leucocyte-endothelium } \\
\text { adhesion, trans-migration }\end{array}$ \\
\hline & $\mathrm{CD} 49 \mathrm{~b}$ & $\alpha$-Chain of VLA-2 & & & $\begin{array}{l}\text { Firm leucocyte-endothelium } \\
\text { adhesion }\end{array}$ \\
\hline & CD49d & $\alpha$-Chain of VLA-4 & & & $\begin{array}{l}\text { Firm lymphocyte-endothelium } \\
\text { adhesion }\end{array}$ \\
\hline & CD49b/CD29 & VLA-2, integrin $\alpha 2 \beta 1$ & & ECM proteins & \\
\hline & CD49c/CD29 & VLA-3, integrin $\alpha 3 \beta 1$ & & ECM proteins & \\
\hline & CD49d/CD29 & VLA-4, integrin $\alpha 4 \beta 1$ & Lymphocytes, monocytes & $\begin{array}{l}\text { VCAM-1, ECM } \\
\text { proteins }\end{array}$ & \\
\hline \multirow[t]{3}{*}{$\begin{array}{l}\text { Ig-super- } \\
\text { family }\end{array}$} & CD54 & ICAM-1 & $\begin{array}{l}\text { Endothelial cells, lympho- } \\
\text { cytes }\end{array}$ & LFA-1, Mac-1 & $\begin{array}{l}\text { Firm lymphocyte-endothelium } \\
\text { adhesion }\end{array}$ \\
\hline & CD8 & & $\begin{array}{l}\text { Cytotoxic T cells, NK cells } \\
(\alpha \text {-chain) }\end{array}$ & $\begin{array}{l}\text { MHC class I } \\
\text { molecules, }\end{array}$ & $\begin{array}{l}\text { Co-receptor to T-cell receptor, } \\
\text { signal transduction }\end{array}$ \\
\hline & CD44 & & & Hyaluronan & \\
\hline
\end{tabular}

CD, cluster of differentiation; LFA, lymphocyte function associated; Mac-1, macrophage adhesion ligand-1; ICAM-1, intercellular adhesion molecule-1; CR, complement receptor; VLA, very late antigen; ECM, extracellular matrix; VCAM, vascular cell adhesion molecule; NK, natural killer; MHC, major histocompatibility complex..

a pressure ulcer, ranging from 0 (healed) to 17 (worst possible score). The score is a summation of three ulcer parameters, each of which is graded according to increments in severity: (1) length $\times$ width of the ulcer; (2) amount of exudate (none, light, moderate and heavy) and (3) tissue type (necrotic tissue, slough, granulation tissue, epithelial tissue and closed). Observation of the changes in the direction and magnitude of the score over time provides a validated indication regarding wound healing ${ }^{(10)}$. In the present study, measurements were recorded on day 0 (enrolment) and on days 7, 14 and 28 . All pressure ulcer scale for healing tool assessments were performed by a single investigator (M. T.)

\section{Statistical analysis}

Data were analysed utilising SPSS 17 for Windows (Chicago, IL, USA). Differences in baseline data and patient characteristics were assessed by the Wilcoxon and independent $t$ tests for non-parametric and parametric variables, respectively. The response of percentage positive CD (cluster of differentiation) and pressure ulcer severity to treatment was analysed by repeated-measures ANOVA. A $P$ value $<0.05$ was considered significant.

\section{Results}

\section{General characteristics}

A total of forty patients (twenty in each group) were enrolled in the study. There were thirteen males and seven females in the control group and fourteen males and six females in the intervention group. There were no statistically significant differences in patient characteristics between the two groups (Table 3). In addition, there were no significant betweengroup differences in leucocyte counts at any time point between the two groups.

\section{Pressure ulcer severity}

Upon enrolment, the severity of pressure ulcers was similar in the control and intervention groups (9.3 (SD 2.1) v.9.1 (SD 2.8) points, respectively; NS). The pressure ulcer scale for healing score increased significantly over time for the control group $(P<0 \cdot 05)$, but not for the intervention group (Fig. 1).

\section{Measurement of percentage of positive adhesion molecules}

Apart from a higher percentage of positive CD18 lymphocytes in the control group $(24.4$ (SD 27.4) v. 48.1 (SD 38.1)\% in the intervention group; $P<0.05$ ), there were no statistically significant differences in baseline percentage of positive CD

Table 3. Patient characteristics

(Number of patients and mean values and standard deviations)

\begin{tabular}{|c|c|c|c|c|c|}
\hline \multirow[b]{2}{*}{ Characteristics } & \multicolumn{2}{|c|}{ Control group } & \multicolumn{2}{|c|}{$\begin{array}{l}\text { Intervention } \\
\text { group }\end{array}$} & \multirow[b]{2}{*}{$P$} \\
\hline & Mean & SD & Mean & SD & \\
\hline$n$ & \multicolumn{2}{|c|}{20} & \multicolumn{2}{|c|}{20} & \\
\hline Age (years) & $53 \cdot 1$ & $19 \cdot 3$ & 49.3 & $20 \cdot 7$ & NS \\
\hline BMI $\left(\mathrm{kg} / \mathrm{m}^{2}\right)$ & $32 \cdot 1$ & 9.9 & 28.3 & $4 \cdot 8$ & NS \\
\hline ICU length of stay $(\mathrm{h})$ & 507 & 217.8 & $627 \cdot 2$ & 340.9 & NS \\
\hline APACHE II score & $25 \cdot 7$ & 7 & 23 & $6 \cdot 7$ & NS \\
\hline
\end{tabular}

ICU, intensive care unit; APACHE II, Acute Physiology and Chronic Health Evaluation II. 


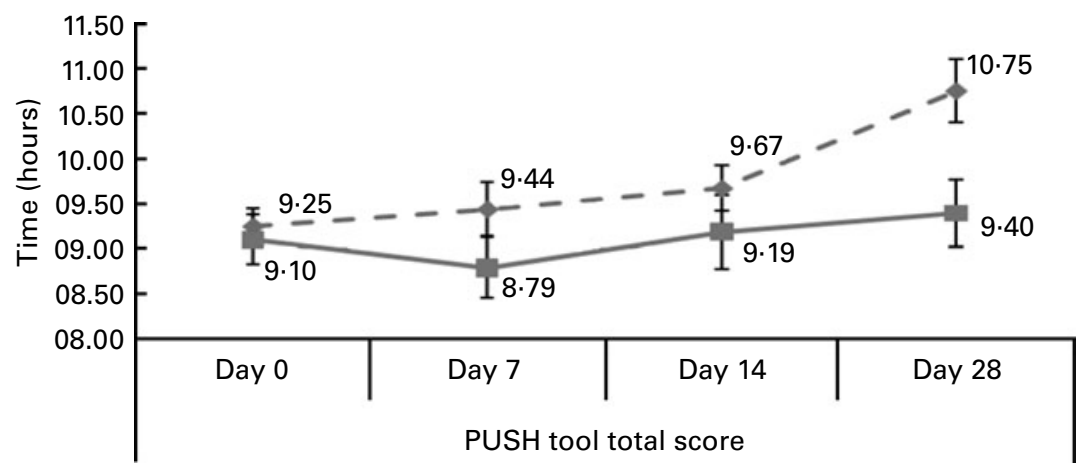

Fig. 1. Changes in the Pressure Ulcer Scale for Healing (PUSH) tool total score between the intervention (- - ) and control (- - $-\cdot)$ groups over time.

between the two groups (Fig. 2). Following 1 week of treatment, percentage of positive CD18 lymphocytes increased to $45 \cdot 2(\mathrm{sD} 33 \cdot 2) \%$ in the intervention group, whereas the control group exhibited a decrease to $33 \cdot 1$ (SD 24.1) \%. This change in percentage of positive $\mathrm{CD}$ was statistically significant ( $P<0.05$; Fig. 2). By day 14, the lymphocyte CD18 levels of the two groups converged. Repeated-measures ANOVA also demonstrated a statistically significant interaction between the effects of time and treatment allocation on lymphocyte CD18 $(P<0 \cdot 05)$. The percentages of positive CD11a lymphocytes and CD49b granulocytes were similar between the two groups at baseline and increased significantly in response to treatment in the intervention, but not control group $(P<0.05$, group $\times$ time interaction effect). Lymphocyte CD11a increased from 54.4 (SD $40 \cdot 3$ ) \% (day 0) to $70 \cdot 8$ (SD 39) $\%$ (day 7) in the intervention group $v .61$ (SD 43) \% (day 0 ) to 68.7 ( $\mathrm{sD} \mathrm{35.6) \% (day} \mathrm{7)} \mathrm{in} \mathrm{patients} \mathrm{receiving} \mathrm{the} \mathrm{control}$ formula $(P<0.05$; Fig. 3$)$. The mean percentage of positive CD49b granulocytes increased significantly in the intervention group from 48.7 (SD 45.7 ) \% on day 0 to 83.3 (SD 34.4 ) \% on day $14(P<0.05)$, and decreased in the control group from $47 \cdot 3$ (sD $46 \cdot 8) \%$ to $39 \cdot 1$ (SD $42 \cdot 1) \%(P<0 \cdot 05$; Fig. 4 ).

The intervention group also showed a trend towards an increase in the percentage of positive CD11b lymphocytes and CD18 granulocytes over $14 \mathrm{~d}$ of treatment $(P=0.06$ in both cases). There were no significant differences in CD44, CD54, CD49d or CD8 between the groups over the study period.

\section{Discussion}

In the present study, we have shown that patients with pressure ulcers who received an $n$-3-LC-PUFA- and micronutrientenriched nutritional formula showed an increase in the percentage of positive lymphocyte and granulocyte adhesion molecules. These patients also had significantly less progression of existing pressure ulcers as compared to patients receiving an iso-nitrogenous, nutrient-sufficient formula.

Critical illness confers susceptibility to the development and persistence of pressure ulcers. Indeed, a significant proportion of ICU patients will develop pressure ulcers, in addition to those who present with these lesions on admission. A recent study which reviewed the literature for the period 2000-5, focusing on the prevalence and incidence of pressure ulcers in intensive care patients, found that while there were variations in intensive care settings ranging from $4 \%$ in Denmark to $49 \%$ in Germany, the overall incidence ranged from 38 to $124 \%{ }^{(11)}$. These pressure ulcers are associated with increased length of hospitalisation and healthcare costs ${ }^{(12)}$

The recruitment of leucocytes from the bloodstream to the tissues, a process essential for wound healing, critically requires the expression and function of CAM. The selectins are involved in the initial phase of inflammatory process, by enabling the rolling of leucocytes to resist the shear force of the blood stream. Thereafter, the activation of integrins results in increased affinity of their ligands on endothelial cells, thus ensuring tight binding of leucocytes to the vascular endothelium so that transendothelial leucocyte migration may follow $^{(2)}$. The fact that leucocyte-endothelial adhesion supports tissue repair is evident from rare genetic disorders involving CD18 mutations (leucocyte antigen deficiency), where the lack of this essential leucocyte-endothelium adhesion molecule is associated with chronicity of wounds and impaired healing ${ }^{(13)}$.

CD11a, CD11b, CD18 and CD49b are transmembrane proteins expressed on granulocytes and lymphocytes that form integrin heterodimers. Flow cytometric analysis in the present study revealed an increase in the percentage positive of these adhesion molecules in patients who received the $n$ - 3 fatty acid- and micronutrient-enriched formula, indicating increased activation and function of these molecules. This increase occurred during the first 7-14d, which coincides

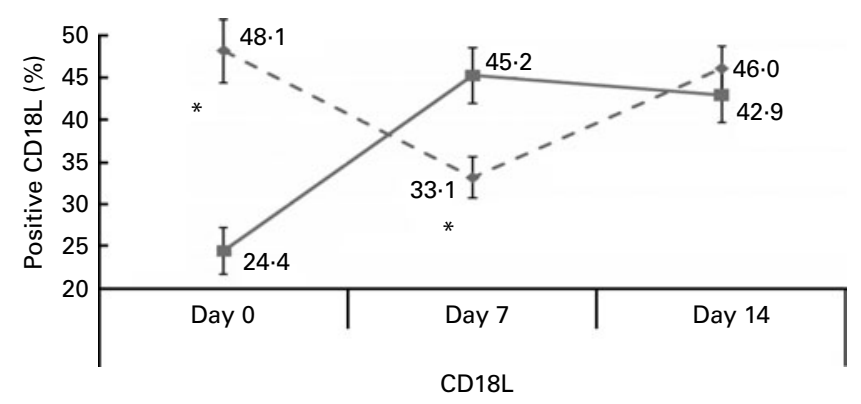

Fig. 2. Changes in percentage of positive CD18 lymphocytes (CD18L) in the control $\left(--_{-}\right)$and intervention ( $-(-)$groups during the study period. * Mean values were significantly different between the control and intervention groups $(P<0.05)$. 


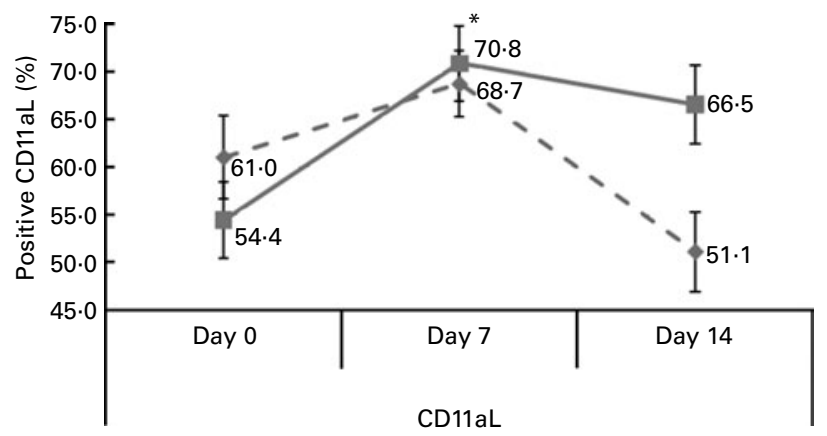

Fig. 3. Percentage of positive CD11a lymphocytes (CD11aL) in the control (- - . $)$ and intervention (- - ) groups as a function of time. * Mean values were significantly different between day 0 and day 7 for the control and intervention groups $(P<0.05)$.

with the inflammatory phase of wound healing. Lymphocyte CD11a/CD18 interacts not only with intracellular adhesion molecule-1 on endothelial cells, but also with keratinocyte intracellular adhesion molecule-1, which is required in the third phase of wound healing, i.e. the repair phase ${ }^{(14)}$. We have also recently shown (M Theilla, B Schwartz, J Cohen, $\mathrm{R}$ Anbar and $\mathrm{P}$ Singer, unpublished results) that patients with pressure ulcers who received the same $n$-3-LC-PUFA- and micronutrient-enriched nutritional formula displayed a significant decrease in C-reactive protein levels as compared to controls. This suggests that this formula may have a dampening effect on acute inflammatory processes. Thus, it is interesting to speculate that the positive effect of the $n$ - 3 fatty acid on preventing pressure ulcer progression could be the result of both an increase in the percentage of positive leucocyte adhesion molecules together with possible attenuation of an excessive inflammatory response.

There have been conflicting results in the literature regarding the effect of fish oil-based nutrition on adhesion molecule expression. $n-3$ has been shown to inhibit TNF- $\alpha$, IL-1 $\beta$ and platelet-activating factor, all of which increase CAM expression/activity so that a reduction in the percentage of positive adhesion molecules would be expected, as demonstrated by Mayer et al. ${ }^{(15)}$ and others ${ }^{(16-19)}$. In addition, $n$-3-LC-PUFA have been shown to reduce the percentage of positive leucocyte adhesion molecules and impaired leucocyte-endothelial interactions ${ }^{(20-22)}$. However, others have described a neutral ${ }^{(23)}$ or enhancing $^{(24)}$ effect of fish oil on percentage of positive adhesion molecules in experimental settings. $n-3$ may inhibit the secretion of TNF- $\alpha$-dependent proteases by circulating leucocytes. Certain proteases cleave membrane CAM, reducing their number (e.g. cleavage of neutrophil CD18 by the lysosomal cysteine proteinase, cathepsin B $)^{(25)}$. Neutrophil CD11b/CD18 also undergo cleavage, but only following extravasation to the skin ${ }^{(26)}$. Thus, even if $n-3$ inhibits NF- $\mathrm{kB} /$ platelet-activating factor/ TNF- $\alpha$-induced percentage positive of CAM, it may reduce their proteolysis to a greater extent, thereby increasing their number, on average. The results of our present study appear to support the positive effect of PUFA on CAM expression in ICU patients and in view of the paucity of results in these patients, additional clinical trials appear to be warranted.
An alternative interpretation of our findings is that the association between increases in the percentage of positive CAM and n-3-LC-PUFA-mediated enhancement of wound healing is not of a causative nature, i.e. was mediated by adhesion-independent mechanism(s). Thus, for example, $n$-3-LC-PUFA are the source of several lipid mediators (e.g. resolvins, lipoxins, epoxyeicosatetraenoic acid) that appear to switch tissue macrophage action from a pro-inflammatory and microbicidal mode to an anti-inflammatory and proresolution mode. Following the initial inflammatory response to tissue injury, some macrophage sub-types leave the area of tissue injury via the lymphatics while others remain in situ to continue the process of tissue repair ${ }^{(25)}$. It is possible, therefore, that locally formed resolvins/protectins and $n$-3-derived PPAR- $\gamma$ agonists may induce a 'wound-healing' phenotype in tissue macrophages. Resolvins such as resolvin D2 have been shown to reduce the trafficking of leucocytes to inflammatory loci and to reduce CD26L and CD18 ${ }^{(17,27)}$. This further inhibits the inflammatory infiltration and initiates the transition to a reparative stage of healing (inflammationresolving type macrophages). Finally, nutritional support with fish oil may prevent hypotension and improve oxygenation in critical illness ${ }^{(28,29)}$, thus maintaining cutaneous blood flow and oxygen supply and facilitating the healing of pressure ulcers.

The major limitation of the present study lies in the fact that we measured only circulating markers. However, immune cells in tissue and plasma do not necessarily reflect the same phenotype and may have differing responsiveness. Mechanistic studies that include the assessment of tissue physiology are clearly necessary in order to determine the mechanism(s) by which $n-3$ fish oil and micronutrients may facilitate the healing of pressure ulcers in ICU patients.

In summary, nutritional support in general may play an important role in the treatment of wound healing and pressure ulcers, and specific nutrients may further modulate the efficacy of nutrition. Our findings suggest that $n$-3-LC-PUFA may have a positive effect on wound healing and that this effect may be mediated by adhesion-dependent mechanisms. We believe that our findings are novel and expand the therapeutic repertoire of fish oil in the critically ill.

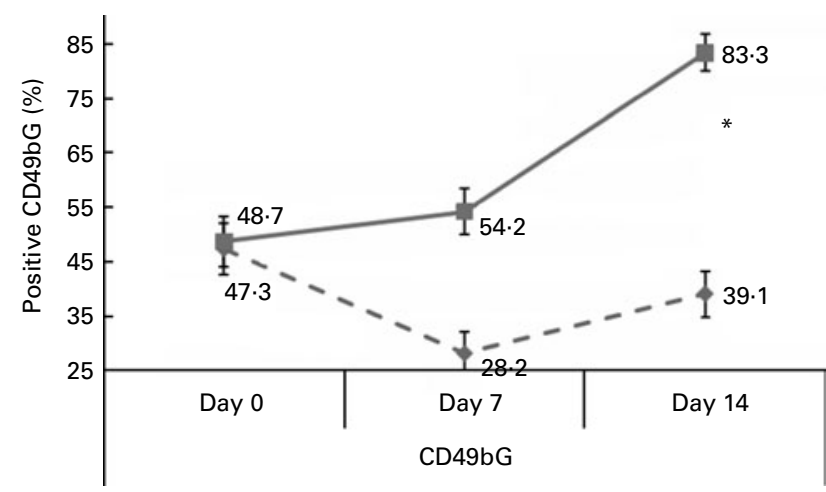

Fig. 4. Percentage of positive CD49b granulocytes (CD49bG) in the control $\left(--_{-}\right)$and intervention (- - ) groups as a function of time. Mean values were significantly higher in the intervention group at day 14 and lower in control group $(P<0.05)$. 


\section{Acknowledgements}

M. T. was responsible for the conception, design, data collection, interpretation of the results, writing and reviewing of the manuscript. B. S., H. S. and R. A. contributed to the conception and design of the research, reviewing of the manuscript and interpretation of the results. Y. Z. and E. R. were responsible for the collection and interpretation of the data. J. C. was responsible for the writing and reviewing of the manuscript and interpretation of the results. P. S. contributed to the conception and design of the research, design, writing and reviewing of the manuscript and interpretation of the results. The authors declare that there is no conflict of interests. No funding was received for this study.

\section{References}

1. Gurtner GC, Werner S, Barrandon Y, et al. (2008) Wound repair and regeneration. Nature 453, 314-321.

2. Cruse JM \& Wang H (2002) Cellular and molecular alterations in spinal cord injury patients with pressure ulcers: a preliminary report. Exp Mol Pathol 72, 124-131.

3. Hulsey TK, O'Neill JA, Neblett WR, et al. (1980) Experimental wound healing in essential fatty acid deficiency. $J$ Pediatr Surg 15, 505-508.

4. Bjerve KS (1989) n-3 Fatty acid deficiency in man. J Intern Med Suppl 731, 171-175.

5. Theilla M, Singer P, Cohen J, et al. (2007) A diet enriched in eicosapentanoic acid, gamma-linolenic acid and antioxidants in the prevention of new pressure ulcer formation in critically ill patients with acute lung injury: a randomized, prospective, controlled study. Clin Nutr 26, 752-757.

6. European Pressure Ulcer Advisory Panel (2010) Pressure ulcer prevention guidelines. www.epuap.org/glprevention. html (accessed March 2010).

7. Knaus WA, Draper EA, Wagner DP, et al. (1985) APACHE II: a severity of disease classification system. Crit Care Med 13, $818-829$.

8. Haisjackl M, Hasibeder W, Klaunzer S, et al. (1990) Diminished reactive hyperemia in the skin of critically ill patients. Crit Care Med 18, 813-818.

9. George-Saintilus E, Tommasulo B, Cal CE, et al. (2009) Pressure ulcer PUSH score and traditional nursing assessment in nursing home residents: do they correlate? J Am Med Dir Assoc 10, 141-144.

10. Thomas DR, Rodeheaver GT, Bartolucci AA, et al. (1997) Pressure ulcer scale for healing: derivation and validation of the PUSH tool. Adv Wound Care 10, 96-101.

11. Shahin ES, Dassen T \& Halfens RJ (2008) Pressure ulcer prevalence and incidence in intensive care patients: a literature review. Nurs Crit Care 13, 71-79.

12. Terekeci H, Kucukardali Y, Top C, et al. (2008) Risk assessment of study of the pressure ulcers in intensive care unit patients. Eur J Int Med 20, 394-397.

13. Kuijpers TW, van Lier RA, Hamann D, et al. (1997) LAD1/ variant. A novel immunodefiency syndrome characterized by dysfunctional beta2 integrins. J Clin Invest 100, 1725-1733.
14. Hughes DA, Pinder AC, Piper Z, et al. (1996) Fish oil supplementation inhibits the expression of major histocompatibility complex class II molecules and adhesion molecules on human monocytes. Am J Clin Nutr 63, 267-272.

15. Mayer K, Merfels M, Muhly-Reinholz M, et al. (2002) Omega3 fatty acids suppress monocyte adhesion to human endothelial cells: role of endothelial PAF generation. Am J Physiol Heart Circ Physiol 283, 811-818.

16. Schaefer MB, Ott J, Mohr A, et al. (2007) Immunomodulation by $n$-3- versus $n$ - 6 -rich lipid emulsions in murine acute lung injury - role of platelet-activating factor receptor. Crit Care Med 35, 544-554.

17. Fukuda S \& Schmid-Schonbein GW (2003) Regulation of CD18 expression on neutrophils in response to fluid shear stress. Proc Natl Acad Sci U S A 100, 13152-13157.

18. Evans BJ, McDowall A, Taylor PC, et al. (2006) Shedding of lymphocyte function-associated antigen-1 (LFA-1) in a human inflammatory response. Blood 107, 3593-3599.

19. Kaufman J, Graf BA, Leung EC, et al. (2001) Fibroblasts as sentinel cells: role of the CD 40-CD 40 ligand system in fibroblast activation and lung inflammation and fibrosis. Chest 120, S53-S55.

20. Sanderson P \& Calder PC (1998) Dietary fish oil diminishes lymphocyte adhesion to macrophage and endothelial cell monolayers. Immunology 94, 79-87.

21. Mayer K, Meyer S, Reinholz-Muhly M, et al. (2003) Short-time infusion of fish oil-based lipid emulsions, approved for parenteral nutrition, reduces monocyte proinflammatory cytokine generation and adhesive interaction with endothelium in humans. J Immunol 171, 4837-4843.

22. Albina JE, Gladden P, Walsh WR, et al. (1993) Detrimental effects of an omega-3 fatty acid-enriched diet on wound healing. JPEN J Parenter Enteral Nutr 17, 519-521.

23. Chiu WC, Wang YC, Chien YW, et al. (2009) Effects of dietary fish oil supplementation on cellular adhesion molecule expression and tissue myeloperoxidase activity in hypercholesterolemic mice with sepsis. J Nutr Biochem 20, 254-260.

24. Bates EJ, Ferrante A, Harvey DP, et al. (1993) Polyunsaturated fatty acids increase neutrophil adherence and integrin receptor expression. J Leukoc Biol 53, 420-426.

25. Schwab JM, Chiang N, Arita M, et al. (2007) Resolvin E1 and protectin D1 activate inflammation-resolution programmes. Nature 447, 869-874.

26. Brooks JD, Milne GL, Yin H, et al. (2008) Formation of highly reactive cyclopentenone isoprostane compounds (A3/J3-isoprostanes) in vivo from eicosapentaenoic acid. $J$ Biol Chem 283, 12043-12055.

27. Spite M, Norling LV, Summers L, et al. (2009) Resolvin D2 is a potent regulator of leukocytes and controls microbial sepsis. Nature 461, 1287-1291.

28. Mancuso P, Whelan J, DeMichele SJ, et al. (1997) Effects of eicosapentaenoic and gamma-linolenic acid on lung permeability and alveolar macrophage eicosanoid synthesis in endotoxic rats. Crit Care Med 25, 523-532.

29. Singer P, Theilla M, Fisher H, et al. (2006) Benefit of an enteral diet enriched with eicosapentaenoic acid and gammalinolenic acid in ventilated patients with acute lung injury. Crit Care Med 34, 1033-1038. 